\title{
Venous gas bubble formation and decompression risk after scuba diving in persons with chronic spinal cord injury and able-bodied controls
}

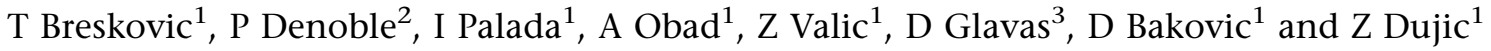 \\ ${ }^{1}$ Department of Physiology, University of Split School of Medicine, Split, Croatia; ${ }^{2}$ Department of Anesthesiology, Center \\ for Hyperbaric Medicine and Environmental Physiology, Duke University Medical Center, Durham, NC, USA and ${ }^{3}$ Department \\ of Internal Medicine, University Hospital Split, Split, Croatia
}

\begin{abstract}
Study design: Prospective study.
Objective: To evaluate the formation of venous gas bubbles following open-sea scuba dives in persons with chronic spinal cord injury $(\mathrm{SCl})$ and in able-bodied diving instructors $(\mathrm{C})$ and to assess the risk for decompression sickness (DCS).

Setting: Field study at the Island of Krk, Croatia.

Methods: Gas bubbles were monitored with an ultrasound scanner $40 \mathrm{~min}$ after surfacing. The probability of DCS $\left(\mathrm{P}_{(\mathrm{DCS})}\right)$ was estimated from the recorded depth-time profile using a decompression model.

Results: Divers completed six dives in 3 days using a modified Bühlmann decompression model, and none developed signs of DCS. Mean $\mathrm{P}_{(\mathrm{DCS})}$ was similar in both groups, $\mathrm{SCl}(0.51 \pm 0.2 \%)$ and $\mathrm{C}$ $(0.64 \pm 0.27 \%)$, and was seen to increase with subsequent dives. Number of bubbles (bubbles per $\mathrm{cm}^{2}$ ) was low in both groups on all 3 days of diving.

Conclusions: We have used the $\mathrm{P}_{(\mathrm{DCS})}$ as a severity index of diving exposure. Overall, the severity of exposure in $\mathrm{SCl}$ subjects was consistent with the range of typical recreational dives, suggesting that the diving profile used is very safe.
\end{abstract}

Spinal Cord (2008) 46, 743-747; doi:10.1038/sc.2008.44; published online 13 May 2008

Keywords: safe diving; decompression sickness; Doppler ultrasound

\section{Introduction}

Scuba diving started as a sport of the exceptionally adventurous and physically fit, but has become a popular recreational activity that attracts both young and old, physically active, as well as sedentary people. All who participate expose themselves to the physical challenges and hazards that are inherent to this activity. The health and fitness requirements required to become certified as a scuba diver vary, but in general appear to have become quite liberal.

The annual incidence of spinal cord injury (SCI) in the United States is approximately 40 cases per million population, or approximately 11000 new cases each year. The number of people in the United States in 2006 who have SCI has been estimated to be between 225000 and 296000 persons. Most injuries occur between the ages of 16 and 30 years. More than half of SCI individuals were employed at

Correspondence: Professor Z Dujic, Department of Physiology, University of Split School of Medicine, Soltanska 2, Split 21000, Croatia.

E-mail: zdujic@bsb.mefst.hr

Received 30 November 2007; revised 25 March 2008; accepted 26 March 2008; published online 13 May 2008 the time of injury and many were actively involved in sports. In the post-injury period, many SCI individuals, mostly those with a lower level of injury, ${ }^{1}$ return to their jobs, lead active lives and some even return to recreation and sports. However, the majority of persons with SCI are inactive and exhibit low levels of cardiovascular fitness, ${ }^{2}$ which is a major independent risk factor for cardiovascular disease and premature mortality. ${ }^{3}$ It appears that the ordinary activities of daily living in SCI are not adequate to maintain cardiovascular fitness. ${ }^{2}$ Historically, individuals with disabilities have been discouraged from pursuing scuba diving by physicians because of both real and perceived risks.

Since the 1990 Americans with Disabilities Act and the 1995 Disability Discrimination Act in Great Britain, dive opportunities for persons with disability have increased. According to a Survey of Scuba Diving for Disabled Divers, over $50 \%$ of British dive clubs were involved in the training of disabled divers between 1998 and 2000. Of the 130 survey respondents who described themselves as disabled, 54 had SCI. One-third of them learned to dive before their injury and two-third learned post injury. ${ }^{4}$ 
There are concerns that diving may further damage the spinal cord, as there is a rare spinal form of decompression sickness (DCS). Neurological abnormalities are presently the most serious decompression related problem in recreational divers requiring treatment. DCS is purportedly due to gas bubbles that form in body tissues during or after decompression. The amount of inert gas taken up by tissues is influenced by dive depth and duration, as well on regional blood perfusion. Two factors required for bubble formation are gas nuclei and supersaturation. The nature of nuclei is unknown, but they are most likely small, gas-filled bubbles attached to the endothelium of blood vessels. These bubbles eventually lodge in the pulmonary circulation. Recently, several reports have indicated that a single dive to depths regularly taken by recreational divers $(\sim 30$ meters of sea water) causes an asymptomatic reduction in cardiovascular function, brachial artery endothelial dysfunction and reduction in right and left ventricle functional indices. ${ }^{5-7}$ Some of these changes were reversed by acute pre-dive administration of antioxidants. ${ }^{7}$ In addition to venous bubble formation during scuba diving, persons with SCI are exposed to other stress factors, such as hypothermia, immersion induced shifts of the peripheral venous blood toward the central vascular compartment, hyperoxia, hemoconcentration and psychological stress.

To date, there is no evidence that the risk of DCS is increased in SCI. The purpose of this study was to compare the occurrence and degree of post-dive venous gas bubbles in divers with disabilities and able-bodied controls.

\section{Methods}

\section{Study population}

A total of 13 divers participated, 6 with chronic SCI and 7 healthy male scuba diving instructors (group C). Demographic and lung function data are shown in Table 1 . The diving instructors composing group $\mathrm{C}$ had years of diving experience, with logged hours of diving between 1000 and 3000. For SCI subjects to be included they had to be at least 5 years out from their SCI and should not have cardiovascular diseases or diabetes. Paraplegia was the result of traffic injury in five subjects. These five became certified divers after becoming paraplegic. One SCI subject was paraplegic due to DCS, and had completed scuba training before injury. Diving

Table 1 Anthropometric characteristics of subjects

\begin{tabular}{lcc}
\hline & $C(\mathrm{n}=7)$ & $S C I(\mathrm{n}=6)$ \\
\hline Age (years) & $38.3(8.3)$ & $36.2(6.7)$ \\
Height/length (cm) & $189(5)$ & $176(11)^{\star}$ \\
Weight (kg) & $94.4(18.1)$ & $69.3(9.1)^{*}$ \\
Waist circumference (cm) & - & $86.6(8.3)$ \\
Body fat index (\%, body fat per kg, BFI) & $15.8(3.6)$ & $19.5(7.3)$ \\
FEV $1 \%$ predicted) & $104.8(8.3)$ & $87.7(15.3)^{*}$ \\
FVC (\% predicted) & $108.5(9.8)$ & $86.4(13.7)^{*}$ \\
\hline
\end{tabular}

Abbreviations: $\mathrm{C}$, control subjects; $\mathrm{FEV}_{1}$, forced expiratory volume in $1 \mathrm{~s}$; FVC, forced vital capacity; SCI, spinal cord injured.

Values are means (s.d.); differences between groups $\left({ }^{*} P<0.05\right)$. experience in the SCI group varied between 1 and 29 years and 5 and $70 \mathrm{~h}$ per year of diving. One of the SCI individuals was mild smoker (10-15 cigarettes a day). At the time of this study, all subjects had a valid medical waiver for diving and were clear of any symptoms of acute illness. Both groups were moderately trained (personal observation). The study methods and potential risks were explained in detail to participants who gave written informed consent before the experiment.

\section{Spirometry}

Spirometry was performed in the seated position. In the group with SCI, height was recorded by self recall, whereas weight was measured using a wheelchair scale with subjects sitting in their wheelchairs. Body fat index was calculated by the Jackson and Pollock three-site measurement method.

\section{Diving protocol}

Divers were equipped with wet diving suits. All dives were performed according to the ZH-L8 ADT Bühlmann protocol, which is shown in Figure 1. The study spanned over 3 days
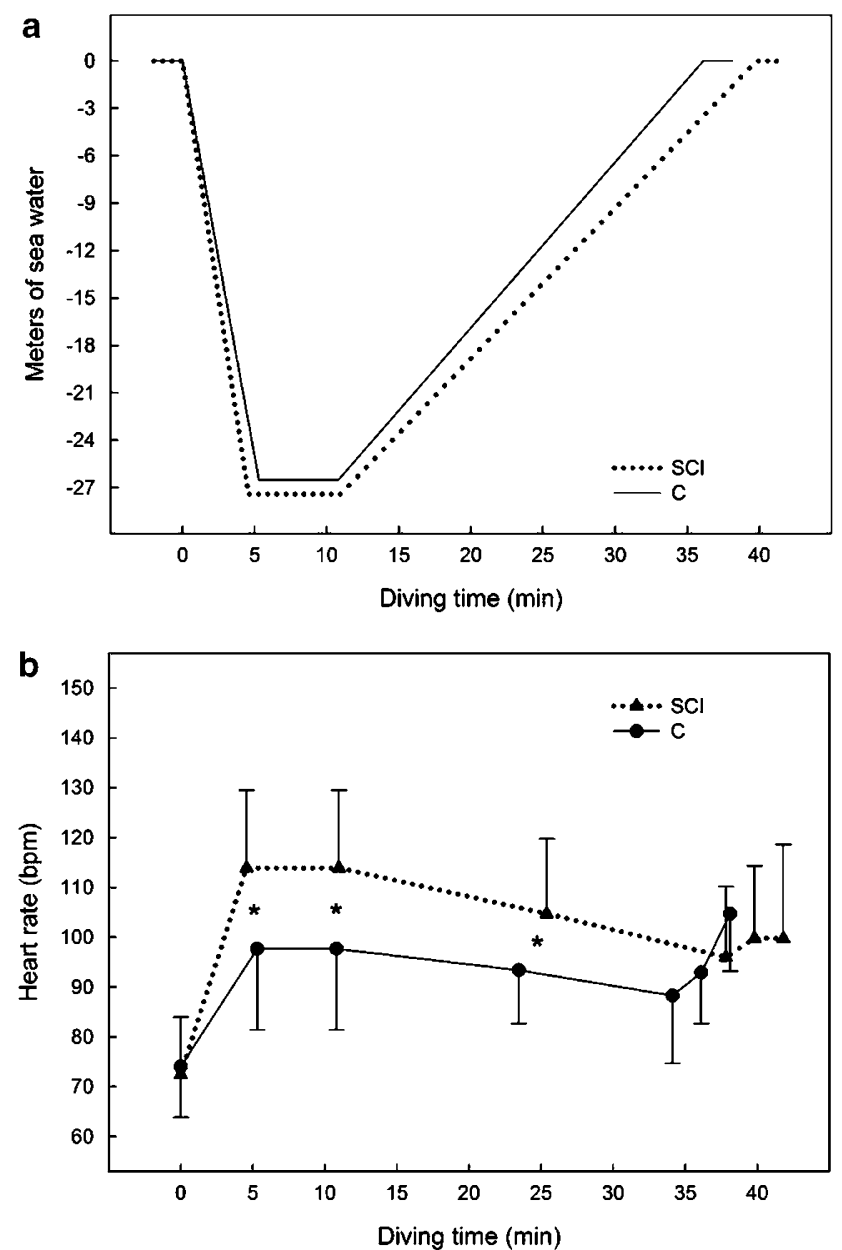

Figure 1 Average diving profile (a) and HR profile (b) for control divers (group $\mathrm{C}$ ) and divers with disability (group $\mathrm{SCl}$ ); differences between groups $\left({ }^{*} P<0.05\right)$. 
during which both groups performed two dives per day (six dives in total). Each SCI subject was accompanied during the dives by an assigned diving instructor. One exception to this rule was a case where one diving instructor monitored two disabled individuals simultaneously, one being a very experienced, independent diver. The dive pairs changed over 3 days. Dive depth was monitored with a dive computer (Galileo, Uwatec, Switzerland) interfaced with personal computer for later verification of the dive profile, sea temperature, and heart rate (HR) of divers. Three divers in each group were equipped with a personal dive computer. During dives, no exercise was performed other than slow swimming. Bottom sea temperatures were $15-17^{\circ} \mathrm{C}$ for all dives, whereas air temperatures varied between 25 and $28^{\circ} \mathrm{C}$.

\section{Post-dive monitoring and bubble analysis}

After completion of each dive, divers were transported to an echocardiographic lab established on the beach $50 \mathrm{~m}$ from the shoreline. Beginning approximately $40 \mathrm{~min}$ after surfacing, with subjects in a supine position, venous gas bubbles were monitored with a Logic Book XP scanner (GE, Milwaukee, WI, USA), using a phase array probe (1.53.3 MHz). Gas bubbles appeared in the right ventricle as high-intensity echoes (Figure 2). Cardiac images were recorded to the computer's hard drive during $60 \mathrm{~s}$ of rest and after two coughs. Coughs were used because such a maneuver significantly increases the number of bubbles detected. ${ }^{8}$ The quality of images obtained in all subjects was very good. We graded bubbles using the method described by Eftedal and Brubakk, ${ }^{9}$ which describes six grades: 0, no bubbles; 1 , occasional bubbles; 2 , at least one bubble every fourth heart cycle; 3 , at least one bubble per cycle; 4, continuous bubbling, at least one bubble per $\mathrm{cm}^{2}$ in all frames and 5, 'white-out', individual bubbles cannot be seen (this grade has been observed in animals only). The grading system has been used extensively in several animal species, as well as in human. After grading, the values were transferred to a linear scale (bubbles per $\mathrm{cm}^{2}$ ) as previously

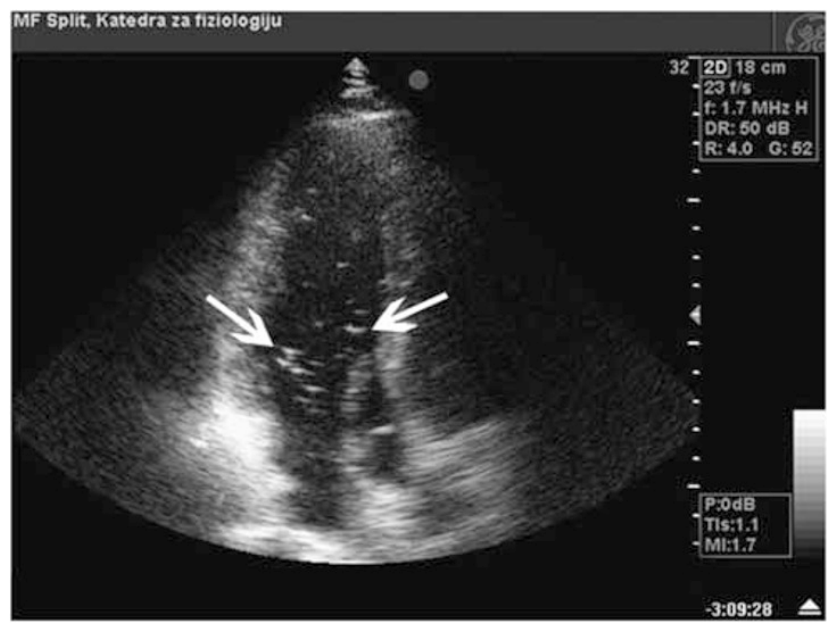

Figure 2 Echocardiographic apical four-chamber view of the heart in one representative control diver with maximal bubble grade (3). Arrows show high-density bubbles in the right heart. described. ${ }^{10}$ Bubbles were assessed twice after each dive and then integrated to give an average bubble number per day. The risk of DCS was estimated from the recorded depth-time profile using a software application developed by W Gerth that implements the decompression model LE1. ${ }^{11}$ The presence of patent foramen ovale in our subjects was checked by observing whether bubbles crossed the atrial septum following two coughs.

\section{Statistics}

The data are presented as mean \pm standard deviation (s.d.). The comparison between groups was done using Student's $t$-test for paired and unpaired samples, using Statistica 7.0 software (Statsoft Inc., Tulsa, OK, USA). The comparison of $\mathrm{P}_{(\mathrm{DCS})}$ in repetitive diving was done using linear regression analysis (SAS by SAS Institute Inc., Cary, NC, USA).

We certify that all applicable institutional and governmental regulations concerning the ethical use of human volunteers were followed during the course of this research.

\section{Results}

SCI patients and able-bodied healthy males were matched for age, but the SCI group was comprised of smaller and lighter individuals (Table 1). Body fat index was similar in SCI and C groups. All 13 divers successfully completed the protocol design comprised of six dives in 3 consecutive days, and none developed any signs/symptoms of DCS. Average bottom depth and time during the dive was $26.9 \pm 2.9 \mathrm{~m}$ and $5.9 \pm 3.9 \mathrm{~min}$, respectively (Figure 1a). Descent speed was $\sim 5.5 \mathrm{~m} \mathrm{~min}^{-1}$, whereas the ascent speed was $\sim 1.2 \mathrm{~m} \mathrm{~min}^{-1}$.

On the surface there was no difference in HR between the groups. During the bottom phase and first half of the ascent phase, SCI patients showed significantly higher $\operatorname{HR}(P=0.01$, $P=0.025$, respectively; Figure $1 \mathrm{~b}$ ).

Of 78 dives among 13 divers, bubbles were detected in all divers. The highest grade detected was three in group $\mathrm{C}$ diver No. II, after his first day of diving. Average linearized bubble grade was similar for days 1 and 3 in both groups, whereas after day 2 diving instructors showed a greater increase in gas bubbles formation (Table 2). Arterialization of bubbles (crossing of the atrial septum or passing through the lung) was not observed in any diver.

The estimated probability of DCS $\left(\mathrm{P}_{(\mathrm{DCS})}\right)$ varied between 0.17 and $1.1 \%$. Only two dives had a $\mathrm{P}_{(\mathrm{DCS})}>1$. The mean $\mathrm{P}_{\text {(DCS) }}$ for SCI subjects $(0.51 \pm 0.2 \%)$ was similar to mean $\mathrm{P}_{\text {(DCS) }}$ for able-bodied divers $(0.64 \pm 0.27 \%$; Figure 3$)$. The $\mathrm{P}_{(\mathrm{DCS})}$ was greater after repeat dives (Figure 4 ).

\section{Discussion}

Peripheral circulation plays a major role in loading inert gases, generally nitrogen, into body tissues during a dive and unloading them after a dive. When a diver is at rest under water, blood will redistribute to the central organs, whereas skeletal muscle will receive little blood. As the visceral organs have a relatively high fat content, and nitrogen is more soluble in fat, more inert gas will be stored there. This 
Table 2 Presents changes in bubble grade per $\mathrm{cm}^{2}$ with and without cough for both experimental groups during the three-day experiment

\begin{tabular}{|c|c|c|c|c|}
\hline \multirow[t]{2}{*}{ Bubble grade (bubbles per $\mathrm{cm}^{2}$ ) } & \multicolumn{2}{|c|}{ No cough } & \multicolumn{2}{|c|}{ Cough } \\
\hline & $C(\mathrm{n}=7)$ & $\mathrm{SCl}(\mathrm{n}=6)$ & $C(\mathrm{n}=7)$ & $\mathrm{SCl}(\mathrm{n}=6)$ \\
\hline 1st day & $0.05(0.19)$ & $0.0(0.01)$ & $0.10(0.26)$ & $0.01(0.02)$ \\
\hline 2nd day & $0.04(0.07)$ & $0.0(0.0)^{\star}$ & $0.07(0.09)$ & $0.0(0.01)^{*}$ \\
\hline 3rd day & $0.0(0.0)$ & $0.0(0.0)$ & $0.01(0.02)$ & $0.1(0.02)$ \\
\hline
\end{tabular}

Abbreviations: $\mathrm{C}$, control subjects; $\mathrm{SCl}$, spinal cord injured.

Values are means (s.d.); differences between groups $\left({ }^{\star} P<0.05\right)$; 1 st day, 2 nd day, 3 rd day present average bubble grade per $\mathrm{cm}^{2}$ for each day.

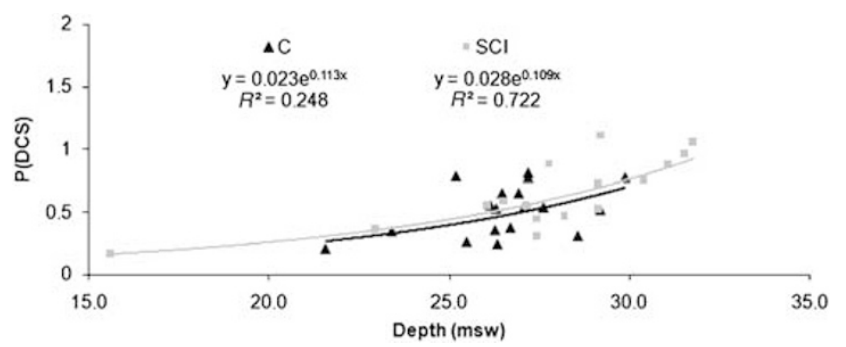

Figure 3 Estimated $\mathrm{P}_{(\mathrm{DCS})}$ for spinal cord injured $(\mathrm{SCl})$ subjects and controls (C) and exponential regression lines.

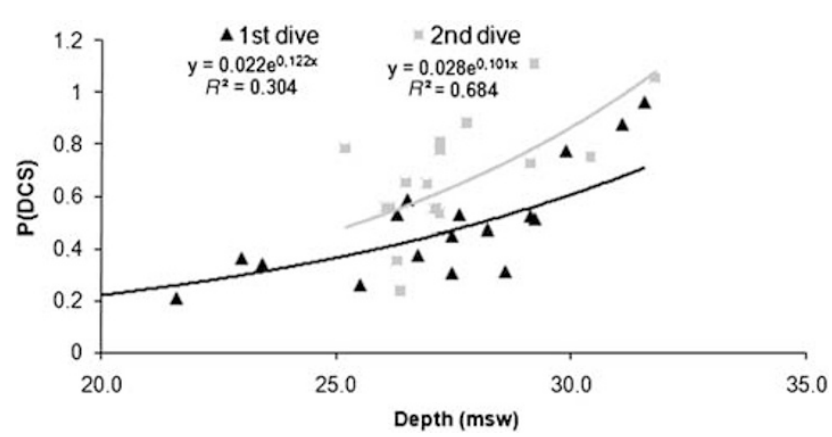

Figure 4 Estimated $P_{(D C S)}$ for first and second dive of day and exponential regression lines.

process is also facilitated by the immersion-induced centralization of peripheral blood. ${ }^{12}$ When a diver performs vigorous exercise underwater, as occurs during most recreational and professional dives, more blood will be directed toward working skeletal muscles and theoretically more inert gas will accumulate in muscle. ${ }^{8}$ However, there is less fat in muscles than in the visceral organs, and therefore the muscle stores less gas. If there are many bubble nuclei in exercising muscle, bubble growth will occur faster in this muscle despite the gas storage capacity being smaller. Circulation changes in paraplegia may affect the risk of DCS. Due to paraplegia, the largest muscle group in the body, the leg, is inactive and takes up less inert gas during a dive. The upper extremities have a higher gas uptake, as swimming is primarily performed by the arms. Based on measured HR changes during the bottom phase and during slow ascent, all subjects performed a moderate level of exercise in this study ( $\sim 30-40 \%$ of the maximal oxygen uptake). Heart rate increased slightly more in SCI subjects, but this was expected, as these subjects have significantly elevated HR values at every submaximal work stage in comparison to control subjects. ${ }^{13}$ In paraplegia, cold exposure during diving may trigger muscle spasticity and a general reaction of autonomic nervous system with hypertension. In addition, SCI patients are at greater risk for disturbed heat balance during exercise in the cold than the able-bodied controls, ${ }^{14}$ and this may adversely affect the outcome of decompression post dive. On the other hand, immersion was shown to be beneficial in alleviating spastic attacks and it has been recommended as a method to prevent spastic attacks. ${ }^{1}$

The present study is the first one known to monitor the occurrence of venous gas bubbles after scuba diving in SCI patients and able-bodied controls. We found that the number of venous gas bubbles was rather low in both groups. The number of bubbles was quantified on a scale from 0 (no bubbles) to 5 bubbles per $\mathrm{cm}^{2}$ ('white out', individual bubbles cannot be seen). ${ }^{9}$ The mean number of bubbles was $\leqslant 0.1$ in all six dives in both SCI and C groups. Although the number of venous bubbles is low, endothelial dysfunction of the brachial artery may still occur, ${ }^{6}$ thus it is difficult to say definitively whether or not recreational diving is a safe sporting activity for SCI subjects.

United States Navy divers with a higher percentage of body fat were reported to have higher risk for DCS, ${ }^{15}$ although we could not confirm this finding. ${ }^{16}$ Furthermore, those who were aerobically more fit produced fewer bubbles after diving. ${ }^{17}$ Body mass index (BMI) was not used in the present study as a marker of obesity in SCI subjects because it has been shown that BMI underestimates obesity. This underestimation is due to potential measurement errors associated with measuring weight and height and because chronic SCI persons generally have greater fat mass and less fat-free mass per unit BMI than age-matched able-bodied control subjects. ${ }^{18,19}$ Greater fat mass may increase inert gas storage in the arms and legs of SCI subjects. Instead of BMI, we used a body fat index and surprisingly found the body fat index was similar between the SCI and C groups. This finding was probably due to the high aerobic fitness level of our SCI subjects (personal observation).

Relatively low bubble grades found after scuba diving in SCI and C subjects in this study may be a result of the diving algorithm employed. The Bühlmann diving algorithm ${ }^{20}$ has been considered safe for many years, and the duration of decompression with this procedure is considerably longer (up to 30-60\%) than what is recommended both by the US Navy, the Norwegian standard tables and the DCIM 
(Canadian) tables. For this study we used an adapted Bühlmann algorithm with a slower speed of descent $\left(\sim 5 \mathrm{~m} \mathrm{~min}^{-1}\right)$ and ascent $\left(\sim 1 \mathrm{~m} \mathrm{~min}^{-1}\right)$. This diving profile is much more conservative than the usual recreational diving profile (descent rate $10 \mathrm{~m} \mathrm{~min}^{-1}$ and ascent rate $9 \mathrm{~m} \mathrm{~min}^{-1}$ ). With a slower compression phase, less inert gas will be accumulated and with a prolonged decompression phase more gas will be eliminated, resulting in reduced DCS risk.

In this study we used the $\mathrm{P}_{(\mathrm{DCS})}$ as an index of diving exposure severity. There was no difference in exposure severity between the two groups. The exposure was more severe on the second dive than on the first dive, suggesting a slightly higher risk. Overall, the severity of exposure was in the range of most recreational dives.

In conclusion, we suggest that chronic SCI subjects in good cardiovascular fitness may engage safely in recreational scuba diving if the modified Bühlmann diving algorithm is used and if they dive in pair with diving instructors. Further studies are needed to investigate the acute and/or long-term effects of diving on cardiovascular function in SCI subjects with lower cardiovascular fitness.

\section{Acknowledgements}

We thank Dr JoAnn A Giaconi (University of California Los Angeles Jules Stein Eye Institute Department of Ophtalmology, Los Angeles, CA, USA) for help with the preparation of the manuscript. We also thank Mr Branko Ravnak, president of IAHD Adriatic International and International Societies Association IAHD Adriatic for their help and support. This study was supported by the Croatian Ministry of Science, Education and Sports, grant nos. 216-2160133-0130 and 2162160133-0330.

\section{References}

1 Steinbruck K, Paeslack V. Analysis of 139 spinal cord injuries due to accidents in water sports. Paraplegia 1980; 18: 86-93.

2 Hoffman MD. Cardiorespiratory fitness and training in quadriplegics and paraplegics. Sports Med 1986; 3: 312-330.

3 Blair SN, Brodney S. Effects of physical inactivity and obesity on morbidity and mortality: current evidence and research issues. Med Sci Sports Exerc 1999; 31: S646-S662.

4 Shelly S, Dowse ML, Bryson P. The Report on the Data from the 1998-2000 Survey of Scuba Diving for Disable Divers. Diving Disease Research Center: Plymouth, 2002.
5 Dujic Z, Obad A, Palada I, Valic Z, Brubakk AO. A single open sea air dive increases pulmonary artery pressure and reduces right ventricular function in professional divers. Eur J Appl Physiol 2006; 97: 478-485.

6 Brubakk AO, Duplancic D, Valic Z, Palada I, Obad A, Bakovic D et al. A single air dive reduces arterial endothelial function in man. J Physiol 2005; 566: 901-906.

7 Obad A, Palada I, Valic Z, Ivancev V, Bakovic D, Wisloff U et al. The effects of acute oral antioxidants on diving-induced alterations in human cardiovascular function. J Physiol 2007; 578: 859-870.

8 Flook V. The effect of exercise on decompression bubbles. A Theoretical Study. In: Mekjavic IB, Tipton MJ, Eiken O (eds). Proceedings of the XXII Annual Scientific Meeting of the European Underwater \& Baromedical Society. Bled, Slovenia, 1997, pp 55-61.

9 Eftedal O, Brubakk AO. Agreement between trained and untrained observers in grading intravascular bubble signals in ultrasonic images. Undersea Hyperb Med 1997; 24: 293-299.

10 Nishi R, Brubakk AO, Eftedal O. Bubble detection. In: Brubakk AO, Neumann TS (eds). Bennet and Elliot's physiology and medicine of diving, 5th edn. WB Saunders: London, 2003, pp 501-529.

11 Gerth WA, Vann RD. Comparison of bubble dynamics and US Navy LE1 models as predictors of altitude decompression sickness. Aviat Space Enviromen Med 1995; 66: A95.

12 Gabrielsen A, Johansen LB, Norsk P. Central cardiovascular pressures during graded water immersion in humans. J Appl Physiol 1993; 75: 581-585.

13 Schneider DA, Sedlock DA, Gass E, Gass G. VO2peak and the gasexchange anaerobic threshold during incremental arm cranking in able-bodied and paraplegic men. Eur J Appl Physiol Occup Physiol 1999; 80: 292-297.

14 Boot CR, Binkhorst RA, Hopman MT. Body temperature responses in spinal cord injured individuals during exercise in the cold and heat. Int J Sports Med 2006; 27: 599-604.

15 Dembert ML, Jekel JF, Mooney LW. Health risk factors for the development of decompression sickness among U.S. Navy divers. Undersea Biomed Res 1984; 11: 395-406.

16 Dujic Z, Duplancic D, Marinovic-Terzic I, Bakovic D, Ivancev V, Valic $\mathrm{Z}$ et al. Aerobic exercise before diving reduces venous gas bubble formation in humans. J Physiol 2004; 555: 637-642.

17 Carturan D, Boussuges A, Vanuxem P, Bar-Hen A, Burnet H, Gardette B. Ascent rate, age, maximal oxygen uptake, adiposity, and circulating venous bubbles after diving. J Appl Physiol 2002; 93: 1349-1356.

18 Jones LM, Legge M, Goulding A. Healthy body mass index values often underestimate body fat in men with spinal cord injury. Arch Phys Med Rehabil 2003; 84: 1068-1071.

19 Spungen AM, Adkins RH, Stewart CA, Wang J, Pierson Jr RN, Waters RL et al. Factors influencing body composition in persons with spinal cord injury: a cross-sectional study. I Appl Physiol 2003; 95: 2398-2407.

20 Buhlmann AA. Computation of low-risk compression. Computation model and results of experimental decompression research. Schweiz Med Wochenschr 1988; 118: 185-197. 